\title{
Benzer Dizilerin Benzer Özellikleri
}

Similar Characteristics of Similar Series

\section{Ecesu DUMAN ${ }^{{ }^{*}}$}

${ }^{1}$ Sivas Bilim ve Sanat Merkezi, Sivas / Türkiye

${ }^{1}$ Sivas Science and Art Center, Sivas / Turkey

*ecesuduman@hotmail.com

${ }^{1}$ ORCID: 0000-0002-1281-463X

\author{
MAKALE BILLGISİ / ARTICLE INFORMATION \\ Geliş Tarihi / Date Received \\ 25.10.2019 \\ Yayın Tarihi / Date Published \\ Aralık / December 2019
}

\section{ATIF / CITE as}

Duman, E. (2019). “Benzer Dizilerin Benzer Özellikleri” / “Similar Characteristics of Similar Series”. bilar: Bilim Armonisi Dergisi, 2 (2): 65-85. doi: 10.37215/bilar.2019257651 


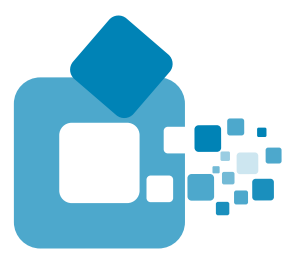

Benzer Dizilerin Benzer Özellikleri"

Similar Characteristics of Similar Series

Matematiğin en önemli konularından biri diziler; dizilerin en dikkat çekenlerinden biri de Fibonacci dizisidir. Bu çalışmanın amacı, Fibonacci dizisine benzer diziler oluşturup bu dizilerin Fibonacci dizisinin özelliklerine benzer özellikleri olup olmadığını araştırmak ve varsa bu özellikleri ispatlamaktır. Bilindiği gibi Fibonacci dizisinde her bir terim, kendisinden önceki iki terimin toplamına eşittir. Bu çalışmada ise, her bir terimin kendinden önceki iki terimden birinin veya her ikisinin 1'den büyük doğal sayılarla çarpılıp sonrasında toplanmasıyla oluşan diziler ele alınmıştır. Böylece üç farklı dizi ailesi oluşturulmuştur. Birinci ailede, her bir terimin kendisinden önceki terimin $\mathrm{k}$ katı $(\mathrm{k}=2,3, \ldots)$ ile ondan önceki terimin toplamına eşit olduğu diziler alındı. İkinci ailede, her bir terimin kendisinden önceki terim ile ondan önceki terimin k katının $(\mathrm{k}=2,3, \ldots)$ toplamına eşit olduğu diziler alındı. Üçüncü ailede ise her bir terimin kendisinden önceki terimin $\mathrm{k}$ katı $(\mathrm{k}=2,3, \ldots)$ ile ondan önceki terimin k katının $(\mathrm{k}=2,3, \ldots)$ toplamına eşit olduğu diziler alındı. Literatürde bu dizilerden bazılarıyla ilgili çalışıldı̆̆ı görülmektedir. Bu çalışmada diğer çalışmalardan farklı olarak, Fibonacci dizisinde geçerli olan 7 özellik temele alındı ve bu özelliklerin benzerleri oluşturulan dizilerde arand1. Her bir dizi ailesinden 2'şer tane olmak üzere 6 dizi üzerinde çalışıldı. Çalışmalar sonunda bu dizilerin de Fibonacci dizisindekilere benzer özellikleri sağladığ1 görülmüştür. Diziler üzerinde 42 özellik elde edilmiş ve bu özellikler ispatlanmıştır. Ayrıca dizilerin oluşum açısından benzerliği olduğu gibi elde edilen özellikler arasında da genellemeye imkân verecek benzerlikler olduğu görülmüştür.
\end{abstract}

Anahtar Sözcükler: Dizi, Fibonacci, Dizi özellikleri, İspat.

\title{
ABSTRACT
}

One of the most important subjects of mathematics is sequences; and one of the most striking series is Fibonacci series. The purpose of this study is to create series similar to Fibonacci series and to investigate whether these series have characteristics similar to those of Fibonacci series; and to prove these characteristics, if any. As is known, in Fibonacci, each term is equal to the sum of the two preceding terms. In this study, the series formed by one of two terms or both terms before each term are multiplied by natural numbers greater than 1 and then summed, were discussed. As a result, three different series families were formed. In the first family, the series were taken in which each term was equal to the sum of $\mathrm{k}$-fold of $(\mathrm{k}=2,3, \ldots)$ the previous term and the two previous terms. In the second family, series were taken in which each term was equal to the sum of the previous term and the $\mathrm{k}$-fold of $(\mathrm{k}=2,3, \ldots)$ the two previous terms. In the third family, series were taken in which each term was equal to the sum of $\mathrm{k}$-fold of $(\mathrm{k}=2,3, \ldots)$ the previous term and $\mathrm{k}$-fold of $(\mathrm{k}=2,3, \ldots)$ the two previous terms. It is seen that some of these series have been studied in the literature. In this study, unlike other studies, seven characteristics which are valid in Fibonacci series were taken as basis and then similar characteristics were searched in the generated series. 6 series were studied by taking 2 from each family. As a result of the studies, it was observed that these series bear similar characteristics to those of Fibonacci series. 42 characteristics were obtained on these series and were proved. In addition, it was observed that there were similarities in terms of formation as well as similarities between the obtained characteristics that would enable generalization.

Keywords: Series, Fibonacci, Characteristics of series, Proof. 


\section{Gíriş}

Günlük hayatta genellikle nesnelerin ya da insanların arka arkaya sıralanmış halini ifade etmek için kullanılan "dizi" kelimesi, matematikte de bu anlamından pek fazla uzaklaşmamıştır. $\mathrm{Bu}$ kez nesneleri sayllar olan bir alanda belli bir ilişkiye veya ortak özelliğe göre sıralanmış sayıları ifade etmek için kullanılmaktadır. Asal sayıların oluşturduğu sıralamalar, beşin katı olan doğal sayıların oluşturduğu sıralamalar, üçgensel sayıların oluşturduğu sıralamalar matematikteki dizi örneklerinden bazılarıdır. Kuşkusuz en ünlü dizilerden biri "Fibonacci Dizisi"dir. Leonardo Fibonacci, (1175-1250) Liber Abaci adlı kitabında bir problem ve problemin çözümünü vererek bu diziden bahsetmiştir. Problem şu şekildedir (Pappas 2007): Bir aylık bir çift tavşan var. Bunlar ancak iki aylık olduklarında yavrulamaya başlayabilir. Bu tavşan çiftin iki aylık olduktan sonra her ay yeni bir çift tavşanı doğduğunu varsayalım. Eğer dünyaya gelen her yeni çift de yukarıda belirtildiği gibi yavrularsa, her ayın başında kaç çift tavşan olur? Bu problem çözüldüğünde ilk aydan itibaren tavşan sayılarının 1, 1, 2, 3, 5, 8, 13,... şeklinde ilerlediği görülür. Biraz daha incelendiğinde her bir sayının kendisinden önceki iki sayının toplamı olduğu görülür. İşte bu sayılar Fibonacci dizisinin terimleridir.

Fibonacci dizisindeki ardışık sayıların çam kozalağı, ayçiçeğinin çekirdek dizilimi, ağaçların dallanma biçimleri gibi doğanın pek çok yerinde insanların karşısına çıkması ve bu dizinin terimlerinin bir önceki terime bölümünün güzelliğin ölçüsü altın orana gittikçe yaklaşması Fibonacci dizisinin büyüsünü arttırmıştır. Pek çok insan bu dizi üzerinde araştırma yapmıştır. Hatta Fibonacci dizisinden esinlenerek Lucas, Pell, Jacobsthal gibi yeni diziler oluşturulmuş ve bunlar üzerinde çalışılmıştır. Köken (2008), çalışmasında Fibonacci ve Jacobsthal dizilerinin bir genelleştirilmesi olarak k-Jacobsthal dizilerinin, Lucas ve Jacobsthal Lucas dizilerinin bir genelleştirilmesi olarak da k-Jacobsthal Lucas dizilerinin tanımları ve özelliklerini vermiştir. Bolat (2008), k-Fibonacci sayılarının tanımından faydalanarak k-Lucas sayıları elde etmiş, bu sayılarla ilgili bazı özellikler ve uygulamalar göstermiştir. Güleç'in (2014) çalışmasında, Pell ve Pell-Lucas sayı dizilerinin özellikleri incelenmiş, bu dizilerin matris dizileri üzerinde durulmuştur. Altun (2016) ise genelleştirilmiş Fibonacci ve Lucas polinomlarında yeni bir aile elde ederek bu aile ile ilgili teorem ve özellikler bulmuştur. $\mathrm{Bu}$ çalışmada Fibonacci dizisinden ilham alarak benzer diziler oluşturulmuştur. Yapılan literatür taramasında bunların matematik dünyasında var olan diziler olduğu görülmüştür. Ancak bu konulardaki çalışmalardan farklı olarak benzer yöntemlerle oluşturulan dizileri hep birlikte, aralarındaki ilişkileri göstererek ve en önemlisi bu dizilerin sağladığı özellikleri bulup ispatlayarak ilerlenmiştir.

Bildiğimiz gibi Fibonacci Dizisi aşa ğıda verildiği gibi her bir terimin kendisinden önceki iki terimin toplamı olarak alınmasıyla oluşmuştur.

\begin{tabular}{|c|c|c|c|c|c|c|c|c|c|c|c|}
\hline$a_{1}$ & $a_{2}$ & $a_{3}$ & $a_{4}$ & $a_{5}$ & $a_{6}$ & $a_{7}$ & $a_{8}$ & $a_{9}$ & $a_{10}$ & $\ldots$ & $a_{n}$ \\
\hline 1 & 1 & 2 & 3 & 5 & 8 & 13 & 21 & 34 & 55 & $\ldots$ & $a_{n-1}+a_{n+2}$ \\
\hline
\end{tabular}

$\mathrm{Bu}$ dizinin pek çok özelliği vardır. Bu ilginç özelliklerden bazıları şu şekildedir (Maksudov ve Veliev 1993):

$$
\begin{array}{ll}
\text { 1. } & a_{1}+a_{2}+a_{3}+\ldots+a_{n}=a_{n+2}-1 \\
\text { 2. } & a_{1}+a_{3}+a_{5}+\ldots+a_{2 n-1}=a_{2 n} \\
\text { 3. } & a_{2}+a_{4}+a_{6}+\ldots+a_{2 n}=a_{2 n+1}-1 \\
\text { 4. } & a_{1}-a_{2}+a_{3}-\ldots+(-1)^{n+1} \cdot a_{n}=(-1)^{n+1} \cdot a_{n-1}+1 \\
\text { 5. } & a_{1}^{2}+a_{2}^{2}+a_{3}^{2}+\ldots+a_{n}^{2}=a_{n} \cdot a_{n+1} \\
\text { 6. } & a_{n+m}=a_{n-1} \cdot a_{m}+a_{n} \cdot a_{m+1} \\
\text { 7. } & a_{2 n}=a_{n+1}^{2}-a_{n-1}^{2}
\end{array}
$$

$\mathrm{Bu}$ çalışmada da Fibonacci dizisinin oluşum özelliğinden ilham alınarak yeni diziler oluşturmaya çalışılmış ve bu dizilerin yukarıda verilen Fibonacci dizisinin 7 temel özelliğinin benzerlerini sağlayıp sağlamadığı üzerinde düşünülmüştür. Temelde 3 dizi ailesi alınmıştır. Kolaylık olması açısından her bir dizi ailesine sadece bu çalışma için geçerli isimler verilmiştir:

- $1 \mathrm{~K}$ Ailesi: Her bir terimin kendisinden önceki terimin $\mathrm{k}$ katı $(\mathrm{k}=2,3, \ldots)$ ile ondan önceki terimin toplanmasıyla oluşan diziler. Literatürde k-bonacci dizileri olarak geçmektedir (Bolat 2008). $a_{0}=0$ ve $a_{1}=1 \mathrm{olmak}$ üzere $a_{n}=\mathrm{k} . a_{n-1}+a_{n-2} . \mathrm{k}=2$ için elde edilen versiyonuna Pell dizisi denilmektedir (Güleç 2014).

- K1 Ailesi: Her bir terimin kendisinden önceki terim ile ondan önceki terimin k katının $(\mathrm{k}=2,3, \ldots)$ toplanmasiyla oluşan diziler. $a_{0}=0$ ve $a_{1}=1$ olmak üzere $a_{n}=a_{n-1}+\mathrm{k} \cdot a_{n-2} \cdot \mathrm{k}=2$ için elde edilen versiyonuna Jacobsthal dizisi denilmektedir (Köken, 2008).

- KK Ailesi: Her bir terimin kendisinden hemen önceki iki terimin toplamının k katı olan diziler. $a_{0}=0$ ve $a_{1}=1$ olmak üzere $a_{n}=$ k. $\left(a_{n-1}+a_{n-2}\right)$

\subsection{Amaç}

$\mathrm{Bu}$ çalışmanın amacı Fibonacci dizisine benzer diziler oluşturup bu dizilerin Fibonacci dizisinin 
özelliklerine benzer özellikleri olup olmadı̆̆ını araştırmak ve varsa bu özellikleri ispatlamaktır.

\subsection{Problem}

Fibonacci dizisine benzer şekilde oluşturulan dizilerin, Fibonacci dizisinin özelliklerine benzer özellikleri bulunabilir mi?

Çalışmanın alt problemleri şu şekildedir:

- Fibonacci dizisine benzer şekilde oluşturulan dizilerin temel özellikleri nelerdir?

- Fibonacci dizisine benzer şekilde oluşturulan dizilerin, Fibonacci dizisinin özelliklerine benzer özellikleri var mıdır?

- Oluşturulan dizilerin varsa bulunan özellikleri nasıl ispatlanır?

- Oluşturulan dizilerin bulunan özellikleri birbiriyle ilişkili midir?

\subsection{Tanımlar}

Dizi: $X \neq \varnothing$ bir küme olsun. $\mathbb{N}$ doğal sayılar kümesinden X'e tanımlı her fonksiyona bir dizi denir (Argün, Arıkan, Bulut, Halıcıoğlu 2014).

Dizinin Terimi: $\left\{a_{\mathrm{n}}\right\}$ bir dizi olsun. Her $\mathrm{i} \in \mathbb{N}$ için $a_{\mathrm{i}}{ }^{\prime}$ ye dizinin bir terimi ve $a_{\mathrm{n}}{ }^{\prime}$ ye dizinin genel terimi denir (Argün ve diğ., 2014).

Fibonacci Dizisi: $f_{0}=0$ ve $f_{1}=1$ olmak üzere, terimleri $f_{\mathrm{n}+2}=f_{\mathrm{n}+1}+f_{\mathrm{n}}$ şeklinde olan dizidir. Yani her bir terimi kendisinden önceki iki terimin toplamına eşittir.

\section{MATERYAL ve METOT}

Dizilerin hepsinde $a_{0}=0$ ve $a_{1}=1$ başlangıç terimleri alınarak 3 farklı dizi ailesi ele alınmıştır. Oluşturulan dizi ailelerinin her birinde $\mathrm{k}=1$ alındığında klasik Fibonacci dizisi çıkmaktadır. O yüzden her bir dizi ailesi için $k=2$ değeri ve $k=3$ değeri alınarak elde edilen dizilerin özellikleri bulunmuştur. Özellikler bulunmaya çalışılırken öncelikle Fibonacci dizisinin özellikleri iyice incelenmiştir. Yeni dizilerde her bir özelliğin nasıl değişebileceğiyle ilgili tahminlerde bulunularak ilk başlarda deneme-yanılmalarla bazı özellikler bulunmuştur. İspatlara geçildikten sonra özelliklerin nasıl oluştuğu daha iyi anlaşıldı ğ için geri kalan özelliklerde deneme-yanılma kullanılmamıştır. Her bir dizinin temel oluşum özellikleri kullanılarak özellikler daha rahat ortaya çıkarılmıştır. İspatlarda ise daha çok doğrudan ispat yöntemi kullanılmak üzere yer yer tümevarımla ispat yöntemi de kullanılmıştır. İspatlar sırasında genellikle ilgili dizinin temel oluşum özelliği kullanılmıştır ve bazı yerlerde "[]" içinde açıklama eklenmiştir.

\section{BULGULAR}

Bu bölümde öncelikle her bir dizi ailesinin nasıl oluştuğundan kısaca bahsedilmiş, bu dizilerden elde edilebilecek sabit oranlardan bahsedilmiştir. Bu kısımlar çalışmanın temel konusu olan özellikler kısmının daha detaylı verilebilmesi için ispatları yapılmadan özet geçilmiştir. Daha sonra da her bir dizi ailesinde $\mathrm{k}=2$ ve $\mathrm{k}=3$ değerleri için elde edilen dizilerin Fibonacci dizisindekilere benzer özellikleri bulunmuş ve ispatlanmıştır.

\subsection{KDizi Ailesi ve Özellikleri}

Bilindiği gibi Fibonacci dizisinde her bir terim kendinden önceki terime bölümü altın orana yaklaşır. Burada ikinci dereceden denklemleri kullanarak $a_{n}=\mathrm{k} . a_{n-1}+a_{n-2}$ şeklindeki diziler için de benzer şekilde her bir terimin kendinden önceki terime bölümünün hangi sayılara yaklaşacağı araştırılırsa aşağıdaki sonuçlar elde edilir:

$\mathrm{k}=1$ için Altın Oran $=\frac{1+\sqrt{5}}{2} \mathrm{k}=2$ için Gümüş Oran $=\frac{2+\sqrt{8}}{2} \mathrm{k}=3$ için Bronz Oran $=\frac{3+\sqrt{13}}{2} \mathrm{k}=\mathrm{n}$ için Sabit Oran $=\frac{n+\sqrt{n^{2}+4}}{2}$

\subsubsection{K Dizi Ailesindeki k=2 Dizisinin Özellikleri}

\begin{tabular}{|c|c|c|c|c|c|c|c|c|c|c|c|}
\hline$a_{1}$ & $a_{2}$ & $a_{3}$ & $a_{4}$ & $a_{5}$ & $a_{6}$ & $a_{7}$ & $a_{8}$ & $a_{9}$ & $a_{10}$ & $\ldots$ & $a_{n}$ \\
\hline 1 & 2 & 5 & 12 & 29 & 70 & 169 & 408 & 985 & 2378 & $\ldots$ & $2 . a_{n-1}+a_{n-2}$ \\
\hline
\end{tabular}

1) $a_{1}+a_{2}+a_{3}+\ldots+a_{n-1}+a_{n}=\frac{a_{n+1}+a_{n}-1}{2}$

İspat: $a_{1}+a_{2}+a_{3}+\ldots+a_{n-1}+a_{n}$ 


$$
\begin{aligned}
& =a_{1}+\left(\frac{a_{3}-a_{1}}{2}\right)+\left(\frac{a_{4}-a_{2}}{2}\right)+\left(\frac{a_{5}-a_{3}}{2}\right)+\left(\frac{a_{6}-a_{4}}{2}\right)+\ldots+\left(\frac{a_{n}-a_{n-2}}{2}\right)+\left(\frac{a_{n+1}-a_{n-1}}{2}\right) \\
& =a_{1}+\frac{a_{3}}{2}-\frac{a_{1}}{2}+\frac{a_{4}}{2}-\frac{a_{2}}{2}+\frac{a_{5}}{2}-\frac{a_{3}}{2}+\frac{a_{6}}{2}-\frac{a_{4}}{2}+\ldots+\frac{a_{n}}{2}-\frac{a_{n-2}}{2}+\frac{a_{n+1}}{2}-\frac{a_{n-1}}{2} \\
& =\frac{a_{1}}{2}-\frac{a_{2}}{2}+\frac{a_{n}}{2}+\frac{a_{n+1}}{2}=\frac{a_{n+1}+a_{n}-1}{2} \quad\left[a_{1}=1 \text { ve } a_{2}=2 \text { alınarak düzenlendi. }\right]
\end{aligned}
$$

2) $a_{1}+a_{3}+a_{5}+\ldots+a_{2 n-1}=\frac{a_{2 n}}{2}$

İspat: $a_{1}+a_{3}+a_{5}+\ldots+a_{2 n-3}+a_{2 n-1}$

$$
\begin{aligned}
& =a_{1}+\left(\frac{a_{4}-a_{2}}{2}\right)+\left(\frac{a_{6}-a_{4}}{2}\right)+\left(\frac{a_{8}-a_{6}}{2}\right)+\ldots+\left(\frac{a_{2 n-2}-a_{2 n-4}}{2}\right)+\left(\frac{a_{2 n}-a_{2 n-2}}{2}\right) \\
& =a_{1}+\frac{a_{4}-a_{2}+a_{6}-a_{4}+a_{8}-a_{6}+\ldots+a_{2 n-2}-a_{2 n-4}+a_{2 n}-a_{2 n-2}}{2} \\
& =a_{1}+\frac{a_{2 n-2}-a_{2}}{2}=\frac{a_{2 n}+2 a_{1}-a_{2}}{2}=\frac{a_{2 n}}{2} \quad\left[a_{1}=1 \text { ve } a_{2}=2 \text { olduğundan }\right]
\end{aligned}
$$

3) $a_{2}+a_{4}+a_{6}+\ldots+a_{2 n}=\frac{a_{2 n+1}-1}{2}$

İspat: 1 . özellikte $\mathrm{n}$ yerine $2 \mathrm{n}$ yazılırsa;

$$
a_{1}+a_{2}+a_{3}+\ldots+a_{2 n}=\frac{a_{2 n+1}+a_{2 n}-1}{2}
$$

2. özellikten;

$$
a_{1}+a_{3}+a_{5}+\ldots+a_{2 n-1}=\frac{a_{2 n}}{2}
$$

$\left({ }^{*}\right)$ eşitliğinden $\left({ }^{* *}\right)$ eşitliği taraf tarafa çıkarılırsa aşağıdaki eşitlikler elde edilir:

$$
a_{2}+a_{4}+a_{6}+\ldots+a_{2 n}=\frac{a_{2 n+1}+a_{2 n}-1}{2}-\frac{a_{2 n}}{2}=\frac{a_{2 n+1}-1}{2}
$$

4) $a_{1}-a_{2}+a_{3}-\ldots+(-1)^{n+1} \cdot a_{n}=\frac{(-1)^{n}\left[a_{n}-a_{n+1}\right]+1}{2}$

İspat: 2. ve 3. özelliklerden iki eşitlik yazılır ve taraf tarafa çikarılır:

$$
\begin{aligned}
& a_{1}+a_{3}+a_{5}+\ldots+a_{2 n-1}=\frac{a_{2 n}}{2} \\
& a_{2}+a_{4}+a_{6}+\ldots+a_{2 n}=\frac{a_{2 n+1}-1}{2} \\
& a_{1}-a_{2}+a_{3}-a_{4} \ldots+a_{2 n-1}-a_{2 n}=\frac{a_{2 n}-a_{2 n+1}+1}{2}
\end{aligned}
$$

Bu eşitlikte dikkat edilirse çift indisli terimlerin negatif, tek indisli terimlerin pozitif işaretli olduğu görülür. Bunlara dikkat edilerek ve $2 \mathrm{n}$ yerine $\mathrm{n}$ alınarak son eşitlik tekrar düzenlenirse: 


$$
a_{1}-a_{2}+a_{3}-\ldots+(-1)^{n+1} \cdot a_{n}=\frac{(-1)^{n}\left[a_{n}-a_{n+1}\right]+1}{2}
$$

5) $a_{1}^{2}+a_{2}^{2}+a_{3}^{2}+\ldots+a_{n}^{2}=\frac{a_{n} \cdot a_{n+1}}{2}$

İspat: $a_{1}^{2}+a_{2}^{2}+a_{3}^{2}+a_{4}^{2}+\ldots+a_{n-1}^{2}+a_{n}^{2}$

$$
\begin{aligned}
& =a_{1}^{2}+a_{2} \cdot a_{2}+a_{3} \cdot a_{3}+a_{4} \cdot a_{4}+\ldots+a_{n-1} \cdot a_{n-1}+a_{n} \cdot a_{n} \\
& =a_{1}^{2}+a_{2} \cdot\left(\frac{a_{3}-a_{1}}{2}\right)+a_{3} \cdot\left(\frac{a_{4}-a_{2}}{2}\right)+a_{4} \cdot\left(\frac{a_{5}-a_{3}}{2}\right)+\ldots+a_{n-1} \cdot\left(\frac{a_{n}-a_{n-2}}{2}\right)+a_{n} \cdot\left(\frac{a_{n+1}-a_{n-1}}{2}\right) \\
& =a_{1}^{2}+\frac{a_{2} \cdot a_{3}}{2}-\frac{a_{1} \cdot a_{2}}{2}+\frac{a_{3} \cdot a_{4}}{2}-\frac{a_{2} \cdot a_{3}}{2}+\frac{a_{4} \cdot a_{5}}{2}-\frac{a_{3} \cdot a_{4}}{2}+\ldots+\frac{a_{n} \cdot a_{n+1}}{2}-\frac{a_{n-1} \cdot a_{n}}{2} \\
& =a_{1}^{2}-\frac{a_{1} \cdot a_{2}}{2}+\frac{a_{n} \cdot a_{n+1}}{2}=\frac{a_{n} \cdot a_{n+1}}{2} \quad\left[a_{1}=1 \text { ve } a_{2}=2 \text { yerine alind } 1 .\right]
\end{aligned}
$$

6) $a_{n+m}=a_{n-1} \cdot a_{m}+a_{n} \cdot a_{m+1}$

İspat: m’ye göre tümevarım yapalım.

$\mathrm{m}=1$ için doğru mu? $a_{n+1}=a_{n-1} \cdot a_{1}+a_{n} \cdot a_{1+1} \rightarrow a_{n+1}=a_{n-1}+2 a_{n} \quad$ doğrudur.

$\mathrm{m}=\mathrm{k}$ için doğru olsun: $\quad a_{n+k}=a_{n-1} \cdot a_{k}+a_{n} \cdot a_{k+1}$

$\mathrm{m}=\mathrm{k}+1$ için de doğru olur $\mathrm{mu}$ ?

$a_{n+(k+1)}=a_{(n+1)+k}=a_{n} \cdot a_{k}+a_{n+1} \cdot a_{k+1} \quad[m=\mathrm{k}$ için doğru demiştik. $]$

$=a_{n} \cdot a_{k}+\left(2 \cdot a_{n}+a_{n-1}\right) \cdot a_{k+1} \quad$ [Dizinin özelliğginden $]$

$=a_{n} \cdot a_{k}+2 \cdot a_{n} \cdot a_{k+1}+a_{n-1} \cdot a_{k+1}$

$=a_{n} \cdot\left(a_{k}+2 \cdot a_{k+1}\right)+a_{n-1} \cdot a_{k+1}$

$=a_{n} \cdot a_{k+2}+a_{n-1} \cdot a_{k+1}$

$\mathrm{m}=\mathrm{k}+1$ için doğru olduğunu göstermiş olur. Öyleyse özellik, dizinin tüm terimleri için geçerlidir.

7) $a_{2 n}=\frac{\left(a_{n+1}^{2}-a_{n-1}^{2}\right)}{2}$

İspat: 6. özellik: $\quad a_{n+m}=a_{n-1} \cdot a_{m}+a_{n} \cdot a_{m+1} \quad$ idi. Burada $m=\mathrm{n}$ alalım:

$a_{n+n}=a_{n-1} \cdot a_{n}+a_{n} \cdot a_{n+1}$

$a_{2 n}=a_{n-1} \cdot\left(\frac{a_{n+1}-a_{n-1}}{2}\right)+\left(\frac{a_{n+1}-a_{n-1}}{2}\right) \cdot a_{n+1} \quad$ [Dizinin özelliğinden $]$

$=\frac{a_{n-1} \cdot a_{n+1}-a_{n-1}^{2}+a_{n+1}^{2}-a_{n-1} \cdot a_{n+1}}{2}=\frac{a_{n+1}^{2}-a_{n-1}^{2}}{2}$ elde edilir. 


\subsubsection{K Dizi Ailesindeki k=3 Dizisinin Özellikleri}

\begin{tabular}{|c|c|c|c|c|c|c|c|c|c|c|}
\hline$a_{1}$ & $a_{2}$ & $a_{3}$ & $a_{4}$ & $a_{5}$ & $a_{6}$ & $a_{7}$ & $a_{8}$ & $a_{9}$ & $\ldots$ & $a_{n}$ \\
\hline 1 & 3 & 10 & 33 & 109 & 360 & 1189 & 3927 & 12970 & $\ldots$ & $3 . a_{n-1}+a_{n-2}$ \\
\hline
\end{tabular}

1) $a_{1}+a_{2}+a_{3}+\ldots+a_{n-1}+a_{n}=\frac{a_{n+1}+a_{n}-1}{3}$

İspat: $a_{1}+a_{2}+a_{3}+\ldots+a_{n-1}+a_{n}$

$$
\begin{aligned}
& =a_{1}+\left(\frac{a_{3}-a_{1}}{3}\right)+\left(\frac{a_{4}-a_{2}}{3}\right)+\left(\frac{a_{5}-a_{3}}{3}\right)+\left(\frac{a_{6}-a_{4}}{3}\right)+\ldots+\left(\frac{a_{n}-a_{n-2}}{3}\right)+\left(\frac{a_{n+1}-a_{n-1}}{3}\right) \\
& =a_{1}+\frac{a_{3}}{3}-\frac{a_{1}}{3}+\frac{a_{4}}{3}-\frac{a_{2}}{3}+\frac{a_{5}}{3}-\frac{a_{3}}{3}+\frac{a_{6}}{3}-\frac{a_{4}}{3}+\ldots+\frac{a_{n}}{3}-\frac{a_{n-2}}{3}+\frac{a_{n+1}}{3}-\frac{a_{n-1}}{3} \\
& =\frac{2 a_{1}}{3}-\frac{a_{2}}{3}+\frac{a_{n}}{3}+\frac{a_{n+1}}{3}=\frac{a_{n+1}+a_{n}-1}{3} \quad\left[a_{1}=1 \text { ve } a_{2}=3\right. \text { alınarak düzenlendi.] }
\end{aligned}
$$

2) $a_{1}+a_{3}+a_{5}+\ldots+a_{2 n-1}=\frac{a_{2 n}}{3}$

İspat: $a_{1}+a_{3}+a_{5}+\ldots+a_{2 n-1}$

$$
\begin{aligned}
& =a_{1}+\left(\frac{a_{4}-a_{2}}{3}\right)+\left(\frac{a_{6}-a_{4}}{3}\right)+\left(\frac{a_{8}-a_{6}}{3}\right)+\ldots+\left(\frac{a_{2 n-2}-a_{2 n-4}}{3}\right)+\left(\frac{a_{2 n}-a_{2 n-2}}{3}\right) \\
& =a_{1}+\frac{a_{4}-a_{2}+a_{6}-a_{4}+a_{8}-a_{6}+\ldots+a_{2 n-2}-a_{2 n-4}+a_{2 n}-a_{2 n-2}}{3} \\
& =a_{1}+\frac{a_{2 n-2}-a_{2}}{3}=\frac{a_{2 n}+3 a_{1}-a_{2}}{3}=\frac{a_{2 n}}{3} \quad\left[a_{1}=1 \text { ve } a_{2}=3 \text { olduğundan }\right]
\end{aligned}
$$

3) $a_{2}+a_{4}+a_{6}+\ldots+a_{2 n}=\frac{a_{2 n+1}-1}{3}$

İspat: 1. özellikte n yerine $2 \mathrm{n}$ yazılıp bu eşitlikten 2. özellik çıkarılsın;

$$
\begin{aligned}
& a_{1}+a_{2}+a_{3}+\ldots+a_{2 n}=\frac{a_{2 n+1}+a_{2 n}-1}{3} \\
& a_{1}+a_{3}+a_{5}+\ldots+a_{2 n-1}=\frac{a_{2 n}}{3} \\
& a_{2}+a_{4}+a_{6}+\ldots+a_{2 n}=\frac{a_{2 n+1}+a_{2 n}-1}{3}-\frac{a_{2 n}}{3}=\frac{a_{2 n+1}-1}{3} \\
& \text { 4) } a_{1}-a_{2}+a_{3}-\ldots+(-1)^{n+1} \cdot a_{n}=\frac{(-1)^{n}\left[a_{n}-a_{n+1}\right]+1}{3}
\end{aligned}
$$

İspat: 2. ve 3. özelliklerden iki eşitlik yazılır ve taraf tarafa çikarılır:

$$
a_{1}+a_{3}+a_{5}+\ldots+a_{2 n-1}=\frac{a_{2 n}}{3}
$$


$a_{2}+a_{4}+a_{6}+\ldots+a_{2 n}=\frac{a_{2 n+1}-1}{3}$

$a_{1}-a_{2}+a_{3}-a_{4} \ldots+a_{2 n-1}-a_{2 n}=\frac{a_{2 n}-a_{2 n+1}+1}{3}$

Bu eşitlikte dikkat edilirse çift indisli terimlerin negatif, tek indisli terimlerin pozitif işaretli olduğu görülür. Bunlara dikkat edilerek ve $2 \mathrm{n}$ yerine $\mathrm{n}$ alınarak son eşitlik tekrar düzenlenirse:

$a_{1}-a_{2}+a_{3}-a_{4}+\ldots+(-1)^{n+1} \cdot a_{n}=\frac{(-1)^{n}\left[a_{n}-a_{n+1}\right]+1}{3}$

5) $a_{1}^{2}+a_{2}^{2}+a_{3}^{2}+\ldots+a_{n}^{2}=\frac{a_{n} \cdot a_{n+1}}{3}$

İspat: $a_{1}^{2}+a_{2}^{2}+a_{3}^{2}+a_{4}^{2}+\ldots+a_{n-1}^{2}+a_{n}^{2}$

$$
\begin{aligned}
& =a_{1}^{2}+a_{2} \cdot a_{2}+a_{3} \cdot a_{3}+a_{4} \cdot a_{4}+\ldots+a_{n-1} \cdot a_{n-1}+a_{n} \cdot a_{n} \\
& =a_{1}^{2}+a_{2} \cdot\left(\frac{a_{3}-a_{1}}{3}\right)+a_{3} \cdot\left(\frac{a_{4}-a_{2}}{3}\right)+a_{4} \cdot\left(\frac{a_{5}-a_{3}}{3}\right)+\ldots+a_{n-1} \cdot\left(\frac{a_{n}-a_{n-2}}{3}\right)+a_{n} \cdot\left(\frac{a_{n+1}-a_{n-1}}{3}\right) \\
& =a_{1}^{2}+\frac{a_{2} \cdot a_{3}}{3}-\frac{a_{1} \cdot a_{2}}{3}+\frac{a_{3} \cdot a_{4}}{3}-\frac{a_{2} \cdot a_{3}}{3}+\frac{a_{4} \cdot a_{5}}{3}-\frac{a_{3} \cdot a_{4}}{3}+\ldots+\frac{a_{n} \cdot a_{n+1}}{3}-\frac{a_{n-1} \cdot a_{n}}{3} \\
& =a_{1}^{2}-\frac{a_{1} \cdot a_{2}}{3}+\frac{a_{n} \cdot a_{n+1}}{3}=\frac{a_{n} \cdot a_{n+1}}{3} \quad\left[a_{1}=1 \text { ve } a_{2}=3 \text { yerine alind } 1 .\right]
\end{aligned}
$$

6) $a_{n+m}=a_{n-1} \cdot a_{m}+a_{n} \cdot a_{m+1}$

İspat: m’ye göre tümevarım yapalım.

$\mathrm{m}=1$ için doğru mu? $\quad a_{n+1}=a_{n-1} \cdot a_{1}+a_{n} \cdot a_{1+1}$

$$
a_{n+1}=a_{n-1}+3 \cdot a_{n} \quad\left[a_{1}=1 \text { ve } a_{2}=3\right] \text { dizinin özelliğinden doğrudur. }
$$

$\mathrm{m}=\mathrm{k}$ için, $\quad a_{n+k}=a_{n-1} \cdot a_{k}+a_{n} \cdot a_{k+1}$ eşitliği doğru olsun.

$\mathrm{m}=\mathrm{k}+1$ için de doğru olur $\mathrm{mu}$ ?

$a_{n+(k+1)}=a_{(n+1)+k}=a_{n} \cdot a_{k}+a_{n+1} \cdot a_{k+1} \quad[m=\mathrm{k}$ için doğru demiştik. $]$

$=a_{n} \cdot a_{k}+\left(3 \cdot a_{n}+a_{n-1}\right) \cdot a_{k+1}=a_{n} \cdot a_{k}+3 \cdot a_{n} \cdot a_{k+1}+a_{n-1} \cdot a_{k+1} \quad$ [Dizinin özelliğginden]

$=a_{n} \cdot\left(a_{k}+3 \cdot a_{k+1}\right)+a_{n-1} \cdot a_{k+1}=a_{n} \cdot a_{k+2}+a_{n-1} \cdot a_{k+1}$

$\mathrm{m}=\mathrm{k}+1$ için doğru olduğunu göstermiş olur. Öyleyse özellik, dizinin tüm terimleri için geçerlidir.

7) $a_{2 n}=\frac{\left(a_{n+1}^{2}-a_{n-1}^{2}\right)}{3}$

İspat: 6. Özellik: $\quad a_{n+m}=a_{n-1} \cdot a_{m}+a_{n} \cdot a_{m+1} \quad$ idi. Burada $m=\mathrm{n}$ alalım: 


$$
\begin{aligned}
& a_{n+n}=a_{n-1} \cdot a_{n}+a_{n} \cdot a_{n+1} \\
& a_{2 n}=a_{n-1} \cdot\left(\frac{a_{n+1}-a_{n-1}}{3}\right)+\left(\frac{a_{n+1}-a_{n-1}}{3}\right) \cdot a_{n+1} \\
& =\frac{a_{n-1} \cdot a_{n+1}-a_{n-1}^{2}+a_{n+1}^{2}-a_{n-1} \cdot a_{n+1}}{3}=\frac{a_{n+1}^{2}-a_{n-1}^{2}}{3} \text { elde edilir. }
\end{aligned}
$$

\subsection{K1 Dizi Ailesi ve Özellikleri}

Fibonacci dizisinde her bir terimin kendinden önceki terime bölümü altın orana yaklaşır. Burada ikinci dereceden denklemleri kullanarak $a_{n}=a_{n-1}+\mathrm{k} . a_{n-2}$ şeklindeki diziler için de benzer şekilde her bir terimin kendinden önceki terime bölümünün hangi sayılara yaklaşacağı araştırılırsa aşağıdaki sonuçlar elde edilir:

$\mathrm{k}=1$ için Altın Oran $=\frac{1+\sqrt{5}}{2} \quad \mathrm{k}=2$ için Sabit Oran $=\frac{1+\sqrt{9}}{2} \quad \mathrm{k}=3$ için Sabit Oran $=\frac{1+\sqrt{13}}{2} \quad \mathrm{k}=\mathrm{n}$ için Sabit Oran $=\frac{n+\sqrt{4 . n+1}}{2}$

\subsubsection{K1 Dizi Ailesindeki k=2 Dizisinin Özellikleri}

\begin{tabular}{|c|c|c|c|c|c|c|c|c|c|c|c|}
\hline$a_{1}$ & $a_{2}$ & $a_{3}$ & $a_{4}$ & $a_{5}$ & $a_{6}$ & $a_{7}$ & $a_{8}$ & $a_{9}$ & $a_{10}$ & $\ldots$ & $a_{n}$ \\
\hline 1 & 1 & 3 & 5 & 11 & 21 & 43 & 85 & 171 & 341 & $\ldots$ & $a_{n-1}+2 . a_{n-2}$ \\
\hline
\end{tabular}

1) $a_{1}+a_{2}+a_{3}+\ldots+a_{n}=\frac{a_{n+2}-1}{2}$

İspat: $a_{1}+a_{2}+a_{3}+\ldots+a_{n-1}+a_{n}$

$$
\begin{aligned}
& =\left(\frac{a_{3}-a_{2}}{2}\right)+\left(\frac{a_{4}-a_{3}}{2}\right)+\left(\frac{a_{5}-a_{4}}{2}\right)+\left(\frac{a_{6}-a_{5}}{2}\right)+\ldots+\left(\frac{a_{n+1}-a_{n}}{2}\right)+\left(\frac{a_{n+2}-a_{n+1}}{2}\right) \\
& =\frac{a_{3}-a_{2}+a_{4}-a_{3}+a_{5}-a_{4}+a_{6}-a_{5}+\ldots+a_{n+1}-a_{n}+a_{n+2}-a_{n+1}}{2} \\
& =\frac{a_{n+2}-a_{2}}{2}=\frac{a_{n+2}-1}{2}\left[a_{1}=1 \text { alınd } 1 .\right]
\end{aligned}
$$

2) 2. $a_{n}=\left\{\begin{array}{l}a_{n+1}+1, n \text { tekse } \\ a_{n+1}-1, n \text { çiftse }\end{array}\right.$

İspat: $\mathrm{n}$ sayısının hem tek sayı hem de çift sayı olma durumları için tümevarımla ispatlayalım. $\mathrm{n}$ tek sayı olduğunda $2 . a_{n}=a_{n+1}+1$ eşitliği, $\mathrm{n}$ çift sayı olduğunda 2. $a_{n}=a_{n+1}-1$ sağlanır mı? Tümevarımın ilk adımında tek ve çift için alınması gereken en küçük sayılardan başladık.

$\mathrm{n}=1$ için doğru mu? $\quad 2 \cdot a_{1}=a_{2}+1 \rightarrow 2 \cdot 1=1+1 \rightarrow a_{1}=a_{2}=1 \quad$ oldu ğundan sağland 1 .

$\mathrm{n}=2$ için doğru mu? $\quad 2 . a_{2}=a_{3}-1 \rightarrow 2.1=3-1 \rightarrow a_{2}=1$ ve $a_{3}=3$ olduğundan sa ğland 1 .

$\mathrm{n}=\mathrm{k}$ tek sayısı için 2. $a_{k}=a_{k+1}+1$ eşitliği, $\mathrm{n}=\mathrm{k}$ çift sayısı için $2 . a_{k}=a_{k+1}-1$ eşitliği sağlansın. $\mathrm{n}=\mathrm{k}+1$ için doğru mu yani k+1 tek sayı iken 2. $a_{k+1}=a_{k+2}+1$ eşitliğ $\mathrm{i}, \mathrm{k}+1$ çift sayı iken $2 . a_{k+1}=a_{k+2}-1$ eşitliğ sağlanır $\mathrm{m} 1$ ?

Öncelikle $n=k+1$ tek sayı olsun.

$2 . a_{k+1}=2 \cdot\left(a_{k+2}-a_{k}\right) \quad$ [Dizinin özelliğinden; $\left.a_{k+2}=a_{k+1}+2 . a_{k}\right]$

$=2 \cdot\left(a_{k+2}-a_{k+1}+1\right) \quad\left[\mathrm{k}+1\right.$ tekse, $\mathrm{k}$ çift olur. Bir önceki adımdan 2. $\left.a_{k}=a_{k+1}-1\right]$ 
$2 \cdot a_{k+1}=2 \cdot a_{k+2}-2 \cdot a_{k+1}+2 \rightarrow 4 \cdot a_{k+1}=2 \cdot a_{k+2}+2 \rightarrow 2 \cdot a_{k+1}=a_{k+2}+1$ sağlanmış olur.

Şimdi $n=k+1$ çift sayı olsun.

2. $a_{k+1}=2 \cdot\left(a_{k+2}-a_{k}\right)$ [Dizinin özelliğinden; $\left.a_{k+2}=a_{k+1}+2 . a_{k}\right]$

$=2 .\left(a_{k+2}-a_{k+1}-1\right) \quad\left[\mathrm{k}+1\right.$ çiftse, $\mathrm{k}$ tek olur. Bir önceki adımdan 2. $\left.a_{k}=a_{k+1}+1\right]$

$2 \cdot a_{k+1}=2 \cdot a_{k+2}-2 \cdot a_{k+1}-2 \rightarrow 4 \cdot a_{k+1}=2 \cdot a_{k+2}-2 \rightarrow 2 \cdot a_{k+1}=a_{k+2}-1$ sağlan mış olur.

O halde özellik dizinin tüm terimlerinde geçerlidir.

3) $a_{1}+a_{2}+a_{3}+\ldots+a_{n}=\left\{\begin{array}{l}a_{n+1}, n \text { tekse } \\ a_{n+1}-1, n \text { çiftse }\end{array}\right.$

İspat: $a_{1}+a_{2}+a_{3}+\ldots+a_{n}=\frac{a_{n+2}-1}{2} \quad[1$. özellikten $]$

$=\left\{\begin{array}{l}\frac{\left(2 . a_{n+1}+1\right)-1}{2}, n \text { tekse } \\ \frac{\left(2 . a_{n+1}-1\right)-1}{2}, n c ̧ i f t s e\end{array}\right.$ [n tekse $\mathrm{n}+1$ çift olur. Buna göre 2. özelliği uyguladık.]

$=\left\{\begin{array}{l}\frac{2 \cdot a_{n+1}+1}{2}, n \text { tekse } \\ \frac{2 \cdot a_{n+1}-2}{2}, n \text { çiftse }\end{array}=\left\{\begin{array}{l}a_{n+1}, n \text { tekse } \\ a_{n+1}-1, n \text { çiftse }\end{array}\right.\right.$

4) $a_{1}+a_{2}+a_{3}+\ldots+a_{n}=\left\{\begin{array}{l}2 \cdot a_{n}-1, n \text { tekse } \\ 2 \cdot a_{n}, n \text { çiftse }\end{array}\right.$

İspat: $a_{1}+a_{2}+a_{3}+\ldots+a_{n}=\left\{\begin{array}{l}a_{n+1}, n \text { tekse } \\ a_{n+1}-1, n \text { çiftse }\end{array} \quad\right.$ [3. özellikten]

$$
=\left\{\begin{array}{l}
2 . a_{n}-1, n \text { tekse } \\
2 . a_{n}, n \text { çiftse }
\end{array} \quad[2 . \text { ö zellikten] }\right.
$$

3. ve 4. özellikler 2. özelliğin sonuçlarıdır. 2. özellik diğer özelliklerin ispatı için yardımcıdır.

5) $a_{1}+a_{3}+a_{5}+\ldots+a_{2 n-1}=\frac{a_{2 n+1}+n-1}{3}$

İspat: $a_{1}+a_{3}+a_{5}+\ldots+a_{2 n-1}=A$ olsun. O halde;

$$
\begin{aligned}
& \left.=\left(\frac{a_{3}-a_{2}}{2}\right)+\left(\frac{a_{5}-a_{4}}{2}\right)+\left(\frac{a_{7}-a_{6}}{2}\right)+\ldots+\left(\frac{a_{2 n+1}-a_{2 n}}{2}\right) \text { [Dizinin özelliğinden; } a_{k+2}=a_{k+1}+2 . a_{k}\right] \\
& =\frac{a_{3}-\left(\frac{a_{3}-1}{2}\right)}{2}+\frac{a_{5}-\left(\frac{a_{5}-1}{2}\right)}{2}+\frac{a_{7}-\left(\frac{a_{7}-1}{2}\right)}{2}+\ldots+\frac{a_{2 n+1}-\left(\frac{a_{2 n+1}-1}{2}\right)}{2} \text { [2. özellikten] } \\
& =\frac{a_{3}+1}{4}+\frac{a_{5}+1}{4}+\frac{a_{7}+1}{4}+\ldots+\frac{a_{2 n+1}+1}{4} \\
& =\frac{a_{3}+a_{5}+a_{7}+\ldots+a_{2 n-1}+a_{2 n+1}+n}{4}=\frac{A-1+a_{2 n+1}+n}{4}\left[a_{3}+a_{5}+\ldots+a_{2 n-1}=A-a_{1}=A-1 \text { olur. }\right]
\end{aligned}
$$


$A=\frac{A-1+a_{2 n+1}+n}{4}$

3. $A=a_{2 n+1}+n-1$

$A=a_{1}+a_{3}+a_{5}+\ldots+a_{2 n-1}=\frac{a_{2 n+1}+n-1}{3}$

6) $a_{2}+a_{4}+a_{6}+\ldots+a_{2 n}=\frac{a_{2(n+1)}-n-1}{3}$

İspat: $a_{2}+a_{4}+a_{6}+\ldots+a_{2 n}=A$ olsun. O halde;

$$
\begin{aligned}
& \left.=\left(\frac{a_{4}-a_{3}}{2}\right)+\left(\frac{a_{6}-a_{5}}{2}\right)+\left(\frac{a_{8}-a_{7}}{2}\right)+\ldots+\left(\frac{a_{2 n+2}-a_{2 n+1}}{2}\right) \text { [Dizinin özelliğinden; } a_{k+2}=a_{k+1}+2 . a_{k}\right] \\
& =\frac{a_{4}-\left(\frac{a_{4}+1}{2}\right)}{2}+\frac{a_{6}-\left(\frac{a_{6}+1}{2}\right)}{2}+\frac{a_{8}-\left(\frac{a_{8}+1}{2}\right)}{2}+\ldots+\frac{a_{2 n+2}-\left(\frac{a_{2 n+2}+1}{2}\right)}{2} \text { [2. özellikten] } \\
& =\frac{a_{4}-1}{4}+\frac{a_{6}-1}{4}+\frac{a_{8}-1}{4}+\ldots+\frac{a_{2 n+2}-1}{4} \\
& =\frac{a_{4}+a_{6}+a_{8}+\ldots+a_{2 n}+a_{2 n+2}+n}{4}=\frac{A-1+a_{2 n+1}-n}{4} \quad\left[a_{4}+a_{6}+\ldots+a_{2 n}=A-a_{2}=A-1 \text { olu r. }\right] \\
& A=\frac{A-1+a_{2 n+2}-n}{4} \\
& \text { 3. } A=a_{2 n+2}-n-1 \\
& \text { 7) } a_{1}-a_{2}+a_{3}-\ldots+(-1)^{n+1} \cdot a_{n}=\frac{(-1)^{n} \cdot\left[a_{n+1}-a_{n+2}\right]+n}{3} \\
& A=a_{4}+a_{6}+a_{8}+\ldots+a_{2 n}=\frac{a_{2(n+1)}-n-1}{3}
\end{aligned}
$$

İspat: 5. ve 6. özelliklerdeki eşitlikleri taraf tarafa çıkaralım:

$$
\begin{aligned}
& a_{1}+a_{3}+a_{5}+\ldots+a_{2 n-1}=\frac{a_{2 n+1}+n-1}{3} \\
& a_{2}+a_{4}+a_{6}+\ldots+a_{2 n}=\frac{a_{2(n+1)}-n-1}{3} \\
& a_{1}-a_{2}+a_{3}-a_{4}+\ldots+a_{2 n-1}-a_{2 n}=\frac{a_{2 n+1}+n-1}{3}-\frac{a_{2(n+1)}-n-1}{3} \\
& =\frac{a_{2 n+1}+n-1-a_{2(n+1)}+n+1}{3}=\frac{a_{2 n+1}-a_{2 n+2}+2 n}{3}
\end{aligned}
$$

Çift terimlerin işaretlerinin negatif, tek terimlerin işaretlerinin pozitif olduğu görülür. Bu göz önünde bulundurularak ve $2 \mathrm{n}$ yerine n alınarak tekrar düzenlersek aşağıdaki eşitlik elde edilir:

$$
a_{1}-a_{2}+a_{3}-a_{4}+\ldots+(-1)^{n+1} \cdot a_{n}=\frac{(-1)^{n} \cdot\left[a_{n+1}-a_{n+2}\right]+n}{3}
$$


8) $a_{1}^{2}+a_{2}^{2}+a_{3}^{2}+\ldots+a_{n}^{2}=\frac{(-1)^{n+1} \cdot \boldsymbol{a}_{n+3}+\mathbf{3} \cdot \boldsymbol{a}_{n+1}^{2}+\boldsymbol{n}}{9}$

İspat: $A=a_{1}^{2}+a_{2}^{2}+a_{3}^{2}+a_{4}^{2}+\ldots+a_{n}^{2}$ olsun. O halde;

$$
\begin{aligned}
& A=a_{1} \cdot a_{1}+a_{2} \cdot a_{2}+a_{3} \cdot a_{3}+a_{4} \cdot a_{4}+\ldots+a_{n-1} \cdot a_{n-1}+a_{n} \cdot a_{n} \\
& =a_{1} \cdot\left(a_{2}-2 \cdot a_{0}\right)+a_{2} \cdot\left(a_{3}-2 \cdot a_{1}\right)+a_{3} \cdot\left(a_{4}-2 \cdot a_{2}\right)+a_{4} \cdot\left(a_{5}-2 \cdot a_{3}\right)+\ldots+a_{n} \cdot\left(a_{n+1}-2 \cdot a_{n-1}\right)
\end{aligned}
$$

[Dizinin tanımından $a_{n+2}=2 a_{n}+a_{n-1} \quad$ aldik.]

$=a_{1} \cdot a_{2}-2 \cdot a_{0} \cdot a_{1}+a_{2} \cdot a_{3}-2 \cdot a_{1} \cdot a_{2}+a_{3} \cdot a_{4}-2 \cdot a_{2} \cdot a_{3}+a_{4} \cdot a_{5}-2 \cdot a_{3} \cdot a_{4}+\ldots+a_{n} \cdot a_{n+1}-2 \cdot a_{n-1} \cdot a_{n}$

$=-2 \cdot a_{0} \cdot a_{1}-a_{1} \cdot a_{2}-a_{2} \cdot a_{3}-a_{3} \cdot a_{4}-\ldots-a_{n-1} \cdot a_{n}+a_{n} \cdot a_{n+1}$

[ $a_{0}=0$ alınırsa ve 2 . özellik uygulanırsa;

$$
\begin{aligned}
& =-\left(\frac{a_{2}+1}{2}\right) \cdot a_{2}-\left(\frac{a_{3}-1}{2}\right) \cdot a_{3}-\left(\frac{a_{4}+1}{2}\right) \cdot a_{4}-\ldots-\left(\frac{a_{n} \mp 1}{2}\right) \cdot a_{n}+\left(\frac{a_{n+1} \pm 1}{2}\right) \cdot a_{n+1} \\
& =-\left(\frac{a_{2}^{2}+a_{3}^{2}+\ldots+a_{n}^{2}}{2}\right)+\left(\frac{-a_{2}+a_{3}-a_{4}+\ldots+(-1)^{n+1} \cdot a_{n}}{2}\right)+\frac{(-1)^{n+1} \cdot a_{n+1}}{2}+\frac{a_{n+1}^{2}}{2}
\end{aligned}
$$

[ $a_{1}=1$ alınırsa ve 7 . özellik uygulanırsa;

$$
\begin{aligned}
& A=-\left(\frac{A-1}{2}\right)+\left(\frac{\frac{(-1)^{n} \cdot\left[a_{n+1}-a_{n+2}\right]+n-3}{3}}{2}\right)+\frac{(-1)^{n+1} \cdot a_{n+1}}{2}+\frac{a_{n+1}^{2}}{2} \\
& 3 . A=\frac{(-1)^{n} \cdot a_{n+1}-(-1)^{n} \cdot a_{n+2}+n-3-\mathbf{3} \cdot(-1)^{n} \cdot a_{n+1}+3 \cdot a_{n+1}^{2}+3}{3} \\
& A=\frac{2 \cdot(-1)^{n+1} \cdot a_{n+1}+(-1)^{n+1} \cdot a_{n+2}+3 \cdot a_{n+1}^{2}+n}{9}
\end{aligned}
$$

[Dizinin tanımindan $a_{n+3}=2 a_{n+1}+a_{n+2}$ alırsak;

$$
A=\frac{(-1)^{n+1} \cdot a_{n+3}+3 \cdot a_{n+1}^{2}+n}{9}
$$

9) $a_{n+m}=2 \cdot a_{n-1} \cdot a_{m}+a_{n} \cdot a_{m+1}$

İspat: m’ye göre tümevarım yapalım.

$\mathrm{m}=1$ için doğru mu? $a_{n+1}=2 \cdot a_{n-1} \cdot a_{1}+a_{n} \cdot a_{1+1} \rightarrow a_{n+1}=2 \cdot a_{n-1}+a_{n} \quad\left[a_{1}=a_{2}=1\right]$ doğrudur

$\mathrm{m}=\mathrm{k}$ için, $\quad a_{n+k}=2 \cdot a_{n-1} \cdot a_{k}+a_{n} \cdot a_{k+1}$ eşitliği doğru olsun.

$\mathrm{m}=\mathrm{k}+1$ için doğru olur $\mathrm{mu}$ ?

$a_{n+(k+1)}=a_{(n+1)+k}=2 \cdot a_{n} \cdot a_{k}+a_{n+1} \cdot a_{k+1} \quad[\mathrm{~m}=\mathrm{k}$ için doğru demiştik. $]$ 


$$
\begin{aligned}
& =2 \cdot a_{n} \cdot a_{k}+\left(a_{n}+2 \cdot a_{n-1}\right) \cdot a_{k+1}=2 \cdot a_{n} \cdot a_{k}+a_{n} \cdot a_{k+1}+2 \cdot a_{n-1} \cdot a_{k+1} \\
& =a_{n} \cdot\left(2 \cdot a_{k}+a_{k+1}\right)+2 \cdot a_{n-1} \cdot a_{k+1}=a_{n} \cdot a_{k+2}+2 \cdot a_{n-1} \cdot a_{k+1}
\end{aligned}
$$

$\mathrm{m}=\mathrm{k}+1$ için doğru olduğunu göstermiş olur. Öyleyse özellik, dizinin tüm terimleri için geçerlidir.

10) $a_{2 n}=\left(a_{n+1}\right)^{2}-\left(2 \cdot a_{n-1}\right)^{2}$

İspat: 9. özellikteki m=n alalım. $\quad a_{n+n}=2 \cdot a_{n-1} \cdot a_{n}+a_{n} \cdot a_{n+1}$

$$
\begin{aligned}
& a_{2 n}=2 \cdot a_{n-1} \cdot a_{n}+a_{n} \cdot a_{n+1} \\
& =2 \cdot a_{n-1} \cdot\left(a_{n+1}-2 \cdot a_{n-1}\right)+\left(a_{n+1}-2 \cdot a_{n-1}\right) \cdot a_{n+1} \quad \text { [Dizinin özelliğginden] } \\
& =2 \cdot a_{n-1} \cdot a_{n+1}-\left(2 \cdot a_{n-1}\right)^{2}+\left(a_{n+1}\right)^{2}-2 \cdot a_{n-1} \cdot a_{n+1}=\left(a_{n+1}\right)^{2}-\left(2 \cdot a_{n-1}\right)^{2}
\end{aligned}
$$

\subsubsection{K1 Dizi Ailesindeki k=3 Dizisinin Özellikleri}

\begin{tabular}{|c|c|c|c|c|c|c|c|c|c|c|}
\hline$a_{1}$ & $a_{2}$ & $a_{3}$ & $a_{4}$ & $a_{5}$ & $a_{6}$ & $a_{7}$ & $a_{8}$ & $a_{9}$ & $\ldots$ & $a_{n}$ \\
\hline 1 & 1 & 4 & 7 & 19 & 40 & 97 & 217 & 508 & $\ldots$ & $a_{n-1}+3 \cdot a_{n-2}$ \\
\hline
\end{tabular}

1) $a_{1}+a_{2}+a_{3}+\ldots+a_{n}=\frac{a_{n+2}-1}{3}$

İspat: $a_{1}+a_{2}+a_{3}+\ldots+a_{n}$

$$
\begin{aligned}
& =\left(\frac{a_{3}-a_{2}}{3}\right)+\left(\frac{a_{4}-a_{3}}{3}\right)+\left(\frac{a_{5}-a_{4}}{3}\right)+\left(\frac{a_{6}-a_{5}}{3}\right)+\ldots+\left(\frac{a_{n+1}-a_{n}}{3}\right)+\left(\frac{a_{n+2}-a_{n+1}}{3}\right) \\
& =\frac{a_{3}-a_{2}+a_{4}-a_{3}+a_{5}-a_{4}+a_{6}-a_{5}+\ldots+a_{n+1}-a_{n}+a_{n+2}-a_{n+1}}{3} \\
& =\frac{a_{n+2}-a_{2}}{3}=\frac{a_{n+2}-1}{3}
\end{aligned}
$$

2) $a_{1}+a_{3}+a_{5}+a_{7}+\ldots+a_{2 n-1}=\frac{3 \cdot a_{2 n+1}-a_{2 n+2}-2}{3}$

3) $a_{2}+a_{4}+a_{6}+\ldots+a_{2 n}=\frac{2 \cdot a_{2 n+2}-3 \cdot a_{2 n+1}+1}{3}$

İspat (2. ve 3. Özellikler): $a_{1}+a_{3}+a_{5}+a_{7}+\ldots+a_{2 n-1}=A \quad a_{2}+a_{4}+a_{6}+\ldots+a_{2 n}=B$

$$
\begin{aligned}
& \left.A=\left(\frac{a_{3}-a_{2}}{3}\right)+\left(\frac{a_{5}-a_{4}}{3}\right)+\left(\frac{a_{7}-a_{6}}{3}\right)+\ldots+\left(\frac{a_{2 n+1}-a_{2 n}}{3}\right) \text { [Dizinin özelliğinden; } a_{k+2}=a_{k+1}+3 . a_{k}\right] \\
& =\frac{a_{3}-a_{2}+a_{5}-a_{4}+a_{7}-a_{6}+\ldots+a_{2 n+1}-a_{2 n}}{3} \\
& =\frac{\left(a_{3}+a_{5}+a_{7}+\ldots+a_{2 n+1}\right)-\left(a_{2}+a_{4}+a_{6}+\ldots+a_{2 n}\right)}{3}
\end{aligned}
$$


$A=\frac{\left(A-1+a_{2 n+1}\right)-(B)}{3} \rightarrow 3 \cdot A=A-B+a_{2 n+1}-1 \rightarrow 2 \cdot A+B=a_{2 n+1}-1$

eşitliği elde edilir. Diğer yandan 1. özellik gereği şu eşitliği yazabiliriz:

$a_{1}+a_{2}+a_{3}+\ldots+a_{2 n}=\frac{a_{2 n+2}-1}{3} \rightarrow A+B=\frac{a_{2 n+2}-1}{3}$

$\left({ }^{*}\right)$ eşitliğinden $\left({ }^{* *}\right)$ eşitliği taraf tarafa çıkarılırsa;

$$
A=a_{2 n+1}-1-\frac{a_{2 n+2}-1}{3}=\frac{3 \cdot a_{2 n+2}-3-a_{2 n+2}+1}{3}=\frac{\mathbf{3} \cdot a_{2 n+1}-a_{2 n+2}-\mathbf{2}}{\mathbf{3}}
$$

bulunur. ${ }^{* *}$ ) eşitliğinde bulunan son eşitliği yerine yazıp B'yi yalnız bırakırız:

$$
\frac{3 \cdot a_{2 n+1}-a_{2 n+2}-2}{3}+B=\frac{a_{2 n+2}-1}{3} \rightarrow B=\frac{a_{2 n+2}-1}{3}-\frac{3 \cdot a_{2 n+1}-a_{2 n+2}-2}{3}=\frac{\mathbf{2} \cdot a_{2 n+2}-\mathbf{3} \cdot \boldsymbol{a}_{2 n+1}+\mathbf{1}}{\mathbf{3}}
$$

4) $a_{1}-a_{2}+a_{3}-\ldots+(-1)^{n+1} \cdot a_{n}=(-1)^{n} \cdot\left[2 \cdot a_{n+1}-a_{n+2}\right]-1$

İspat: 2. ve 3. özelliklerdeki eşitklikleri yazıp taraf tarafa çıkaralım:

$$
\begin{aligned}
& \left(a_{1}+a_{3}+a_{5}+a_{7}+\ldots+a_{2 n-1}\right)=\frac{3 \cdot a_{2 n+1}-a_{2 n+2}-2}{3} \\
& \left(a_{2}+a_{4}+a_{6}+\ldots+a_{2 n}\right)=\frac{2 \cdot a_{2 n+2}-3 \cdot a_{2 n+1}+1}{3} \\
& a_{1}-a_{2}+a_{3}-a_{4}+\ldots+a_{2 n-1}-a_{2 n}=\frac{3 \cdot a_{2 n+1}-a_{2 n+2}-2}{3}-\frac{2 \cdot a_{2 n+2}-3 \cdot a_{2 n+1}+1}{3} \\
& =\frac{3 \cdot a_{2 n+1}-a_{2 n+2}-2-2 \cdot a_{2 n+2}+3 \cdot a_{2 n+1}-1}{3}=\frac{6 \cdot a_{2 n+1}-3 \cdot a_{2 n+2}-3}{3}=2 \cdot a_{2 n+1}-a_{2 n+2}-1
\end{aligned}
$$

Çift indisli terimlerin işareti negatifken, tek indisli terimlerin işaretlerinin pozitiftir. Buna dikkat edilerek ve $2 \mathrm{n}$ yerine $\mathrm{n}$ yazılarak düzenlenirse aşağıdaki eşitlik elde edilir:

$$
a_{1}-a_{2}+a_{3}-a_{4}+\ldots+(-1)^{n+1} \cdot a_{n}=(-1)^{n} \cdot\left[2 \cdot a_{n+1}-a_{n+2}\right]-1
$$

5) $\quad a_{n+m}=3 \cdot a_{n-1} \cdot a_{m}+a_{n} \cdot a_{m+1}$

İspat: m’ye göre tümevarım yapalım.

$\mathrm{m}=1$ için doğru mu? $a_{n+1}=3 \cdot a_{n-1} \cdot a_{1}+a_{n} \cdot a_{1+1} \rightarrow a_{n+1}=3 \cdot a_{n-1}+a_{n} \quad\left[a_{1}=a_{2}=1\right]$ doğrudur

$\mathrm{m}=\mathrm{k}$ için, $\quad a_{n+k}=3 \cdot a_{n-1} \cdot a_{k}+a_{n} \cdot a_{k+1}$ eşitliği doğru olsun.

$\mathrm{m}=\mathrm{k}+1$ için doğru olur $\mathrm{mu}$ ?

$a_{n+(k+1)}=a_{(n+1)+k}=3 \cdot a_{n} \cdot a_{k}+a_{n+1} \cdot a_{k+1} \quad[\mathrm{~m}=\mathrm{k}$ için doğru demiştik. $]$

$=3 \cdot a_{n} \cdot a_{k}+\left(a_{n}+3 \cdot a_{n-1}\right) \cdot a_{k+1}=3 \cdot a_{n} \cdot a_{k}+a_{n} \cdot a_{k+1}+3 \cdot a_{n-1} \cdot a_{k+1}$

$=a_{n} \cdot\left(3 \cdot a_{k}+a_{k+1}\right)+3 \cdot a_{n-1} \cdot a_{k+1}=a_{n} \cdot a_{k+2}+3 \cdot a_{n-1} \cdot a_{k+1}$

$\mathrm{m}=\mathrm{k}+1$ için doğru olduğunu göstermiş olur. Öyleyse özellik, dizinin tüm terimleri için geçerlidir. 
6) $a_{2 n}=\left(a_{n+1}\right)^{2}-\left(3 \cdot a_{n-1}\right)^{2}$

İspat: 5. özellikteki eşitlikte $\mathrm{m}=\mathrm{n}$ alalım. $\quad a_{n+n}=3 \cdot a_{n-1} \cdot a_{n}+a_{n} \cdot a_{n+1}$

$$
\begin{aligned}
& a_{2 n}=3 \cdot a_{n-1} \cdot a_{n}+a_{n} \cdot a_{n+1} \\
& =3 \cdot a_{n-1} \cdot\left(a_{n+1}-3 \cdot a_{n-1}\right)+\left(a_{n+1}-3 \cdot a_{n-1}\right) \cdot a_{n+1} \quad \text { [Dizinin özelliğ inden] } \\
& =3 \cdot a_{n-1} \cdot a_{n+1}-\left(3 \cdot a_{n-1}\right)^{2}+\left(a_{n+1}\right)^{2}-3 \cdot a_{n-1} \cdot a_{n+1}=\left(a_{n+1}\right)^{2}-\left(3 \cdot a_{n-1}\right)^{2}
\end{aligned}
$$

\subsection{KK Dizi Ailesi ve Özellikleri}

Fibonacci dizisinde her bir terim kendinden önceki terime bölümü altın orana yaklaşır. Burada ikinci dereceden denklemleri kullanarak $a_{n}=\mathrm{k}$. $\left[a_{n-1}+a_{n-2}\right]$ şeklindeki diziler için de benzer şekilde her bir terimin kendinden önceki terime bölümünün hangi sayılara yaklaşacağı araştırılırsa aşağıdaki sonuçlar elde edilir:

$\mathrm{k}=1$ için Altın Oran $=\frac{1+\sqrt{5}}{2} \quad \mathrm{k}=2$ için Sabit Oran $=\frac{2+\sqrt{12}}{2} \quad \mathrm{k}=3$ için Sabit Oran $=\frac{3+\sqrt{21}}{2} \quad \mathrm{k}=\mathrm{n}$ için Sabit Oran $=\frac{n+\sqrt{n^{2}+4 . n}}{2}$

\subsubsection{KK Dizi Ailesindeki k=2 Dizisinin Özellikleri}

\begin{tabular}{|c|c|c|c|c|c|c|c|c|c|c|}
\hline$a_{1}$ & $a_{2}$ & $a_{3}$ & $a_{4}$ & $a_{5}$ & $a_{6}$ & $a_{7}$ & $a_{8}$ & $a_{9}$ & $\ldots$ & $a_{n}$ \\
\hline 1 & 2 & 6 & 16 & 44 & 120 & 328 & 896 & 2448 & $\ldots$ & $2 . a_{n-1}+2 . a_{n-2}$ \\
\hline
\end{tabular}

1) $a_{1}+a_{2}+a_{3}+\ldots+a_{n}=\frac{a_{n+2}-a_{n+1}-1}{3}$

İspat: $a_{1}+a_{2}+a_{3}+a_{4}+\ldots+a_{n}$

$$
=\left(\frac{a_{3}}{2}-a_{2}\right)+\left(\frac{a_{4}}{2}-a_{3}\right)+\left(\frac{a_{5}}{2}-a_{4}\right)+\left(\frac{a_{6}}{2}-a_{5}\right)+\ldots+\left(\frac{a_{n+1}}{2}-a_{n}\right)+\left(\frac{a_{n+2}}{2}-a_{n+1}\right)
$$

[Dizinin özelliğinden; $a_{n+2}=2 .\left(a_{n+1}+a_{n}\right)$, dolayısıyla $a_{n}=\frac{a_{n+2}}{2}-a_{n+1}$ olur.]

$=-a_{2}-\frac{a_{3}}{2}-\frac{a_{4}}{2}-\frac{a_{5}}{2}-\ldots-\frac{a_{n}}{2}-\frac{a_{n+1}}{2}+\frac{a_{n+2}}{2}=\frac{-a_{2}-\left(a_{2}+a_{3}+a_{4}+\ldots+a_{n}\right)}{2}-\frac{a_{n+1}}{2}+\frac{a_{n+2}}{2}$

$=\frac{-a_{2}-\left(A-a_{1}\right)}{2}-\frac{a_{n+1}}{2}+\frac{a_{n+2}}{2}$

$A=-\frac{(A+1)}{2}-\frac{a_{n+1}}{2}+\frac{a_{n+2}}{2} \quad\left[a_{1}=1\right.$ ve $a_{2}=2$ olduğundan $]$

$A+\frac{(A+1)}{2}=\frac{a_{n+2}-a_{n+1}}{2} \rightarrow A=\frac{a_{n+2}-a_{n+1}-1}{3}$

2) $a_{1}+a_{3}+a_{5}+\ldots+a_{2 n-1}=\frac{1+2 \cdot\left(a_{2 n}-a_{2 n-1}\right)}{3}$

İspat: $a_{1}+a_{3}+a_{5}+\ldots+a_{2 n-1}$

$$
=a_{1}+2 \cdot\left(a_{1}+a_{2}\right)+2 \cdot\left(a_{3}+a_{4}\right)+\ldots+2 \cdot\left(a_{2 n-2}+a_{2 n-3}\right)
$$


$=a_{1}+2 \cdot\left(a_{1}+a_{2}+a_{3}+a_{4}+\ldots+a_{2 n-2}\right)=a_{1}+2 \cdot\left(\frac{a_{2 n}-a_{2 n-1}-1}{3}\right)$

[1. özellikte $n$ yerine $2 n-2$ aldık.]

$=\frac{1+2 .\left(a_{2 n}-a_{2 n-1}\right)}{3} \quad\left[a_{1}=1\right.$ olarak yerine yazdik. $]$

3) $a_{2}+a_{4}+a_{6}+\ldots+a_{2 n}=\frac{2 \cdot\left(a_{2 n+1}-a_{2 n}-1\right)}{3}$

İspat: $a_{2}+a_{4}+a_{6}+\ldots+a_{2 n}$

$=a_{2}+2 \cdot\left(a_{2}+a_{3}\right)+2 \cdot\left(a_{4}+a_{5}\right)+\ldots+2 \cdot\left(a_{2 n-2}+a_{2 n-1}\right)$

$=a_{2}+2 \cdot\left(a_{2}+a_{3}+a_{4}+a_{5}+\ldots+a_{2 n-1}\right)=a_{2}+2 \cdot\left(\frac{a_{2 n+1}-a_{2 n}-1}{3}-1\right)$

[1. özellikte $n$ yerine $2 n-1$ aldık ve $a_{1}$ değeri 1 'i çıkardık.]

$=\frac{2 .\left(a_{2 n+1}-a_{2 n}-1\right)}{3} \quad\left[a_{2}=2\right.$ olarak yerine yazdık ve düzenledik. $]$

4) $a_{1}-a_{2}+a_{3}-a_{4}+\ldots+(-1)^{n+1} \cdot a_{n}=(-1)^{n+1} \cdot 2 \cdot a_{n-1}+1$

İspat: 2. ve 3. özelliklerdeki eşitlikleri taraf tarafa çıkaralım:

$$
\begin{aligned}
& a_{1}+a_{3}+a_{5}+\ldots+a_{2 n-1}=\frac{1+2 \cdot a_{2 n}-2 \cdot a_{2 n-1}}{3} \\
& a_{2}+a_{4}+a_{6}+\ldots+a_{2 n}=\frac{2 \cdot a_{2 n+1}-2 \cdot a_{2 n}-2}{3} \\
& a_{1}-a_{2}+a_{3}-a_{4}+\ldots+a_{2 n-1}-a_{2 n}=\frac{1+2 \cdot a_{2 n}-2 \cdot a_{2 n-1}}{3}-\frac{2 \cdot a_{2 n+1}-2 \cdot a_{2 n}-2}{3} \\
& =\frac{1+2 \cdot a_{2 n}-2 \cdot a_{2 n-1}-2 \cdot a_{2 n+1}+2 \cdot a_{2 n}+2}{3} \\
& =\frac{4 \cdot a_{2 n}-2 \cdot a_{2 n-1}-\mathbf{2} \cdot a_{2 n+1}+3}{3}=\frac{4 \cdot a_{2 n}-2 \cdot a_{2 n-1}-\mathbf{4} \cdot a_{2 n}-\mathbf{4} \cdot a_{2 n-1}+3}{3} \\
& =\frac{-6 \cdot a_{2 n-1}+3}{3}=\mathbf{1}-\mathbf{2} \cdot a_{2 n-1} \\
& {\left[a_{2 n+1}=2 \cdot a_{2 n}+2 \cdot a_{2 n-1} \mathrm{yazd} 1 \mathrm{k} .\right]}
\end{aligned}
$$

Son eşitlikte tek indisli terimlerin pozitif katsayılı, çift indisli terimlerin negatif katsayılı olduğuna dikkat ederek ve $2 \mathrm{n}$ yerine n yazılarak düzenlenirse aşağıdaki eşitlik elde edilir:

$$
a_{1}-a_{2}+a_{3}-a_{4}+\ldots+(-1)^{n+1} \cdot a_{n}=(-1)^{n+1} 2 \cdot a_{n-1}+1
$$

5) $a_{n+m}=2 \cdot a_{n-1} \cdot a_{m}+a_{n} \cdot a_{m+1}$

İspat: m’ye göre tümevarım yapalım. 
$\mathrm{m}=1$ için doğru mu? $\quad a_{n+1}=2 \cdot a_{n-1} \cdot a_{1}+a_{n} \cdot a_{1+1} \rightarrow a_{n+1}=2 \cdot a_{n-1}+2 \cdot a_{n} \quad\left[a_{1}=1\right.$ ve $\left.a_{2}=2\right]$ doğrudur .

$\mathrm{m}=\mathrm{k}$ için, $\quad a_{n+k}=2 \cdot a_{n-1} \cdot a_{k}+a_{n} \cdot a_{k+1}$ eşitliği doğru olsun.

$\mathrm{m}=\mathrm{k}+1$ için doğru olur $\mathrm{mu}$ ?

$a_{n+(k+1)}=a_{(n+1)+k}=2 \cdot a_{n} \cdot a_{k}+a_{n+1} \cdot a_{k+1} \quad[\mathrm{~m}=\mathrm{k}$ için doğru demiştik. $]$

$=2 \cdot a_{n} \cdot a_{k}+\left(2 \cdot a_{n}+2 \cdot a_{n-1}\right) \cdot a_{k+1}=2 \cdot a_{n} \cdot a_{k}+2 \cdot a_{n} \cdot a_{k+1}+2 \cdot a_{n-1} \cdot a_{k+1}$

$=a_{n} \cdot\left(2 \cdot a_{k}+2 \cdot a_{k+1}\right)+2 \cdot a_{n-1} \cdot a_{k+1}=a_{n} \cdot a_{k+2}+2 \cdot a_{n-1} \cdot a_{k+1}$

$\mathrm{m}=\mathrm{k}+1$ için doğru oldu. Öyleyse özellik, dizinin tüm terimleri için geçerlidir.

6) $a_{2 n}=\frac{\left(a_{n+1}\right)^{2}-\left(2 \cdot a_{n-1}\right)^{2}}{2}$

İspat: 5. özellikteki eşitlikte $\mathrm{m}=\mathrm{n}$ alalım. $\quad a_{n+n}=2 \cdot a_{n-1} \cdot a_{n}+a_{n} \cdot a_{n+1}$

$$
\begin{aligned}
& a_{2 n}=2 \cdot a_{n-1} \cdot\left(\frac{a_{n+1}-2 \cdot a_{n-1}}{2}\right)+\left(\frac{a_{n+1}-2 \cdot a_{n-1}}{2}\right) \cdot a_{n+1} \\
& a_{2 n}=\frac{2 \cdot a_{n-1} \cdot a_{n+1}-\left(2 \cdot a_{n-1}\right)^{2}+\left(a_{n+1}\right)^{2}-2 \cdot a_{n-1} \cdot a_{n+1}}{2} \\
& a_{2 n}=\frac{\left(a_{n+1}\right)^{2}-\left(2 \cdot a_{n-1}\right)^{2}}{2}
\end{aligned}
$$

\subsubsection{KK Dizi Ailesindeki k=3 Dizisinin Özellikleri}

\begin{tabular}{|c|c|c|c|c|c|c|c|c|c|}
\hline$a_{1}$ & $a_{2}$ & $a_{3}$ & $a_{4}$ & $a_{5}$ & $a_{6}$ & $a_{7}$ & $a_{8}$ & $\ldots$ & $a_{n}$ \\
\hline 1 & 3 & 12 & 45 & 171 & 648 & 2457 & 9315 & $\ldots$ & $3 \cdot a_{n-1}+3 \cdot a_{n-2}$ \\
\hline
\end{tabular}

1) $a_{1}+a_{2}+a_{3}+\ldots+a_{n}=\frac{a_{n+2}-2 \cdot a_{n+1}-1}{5}$

İspat: $A=a_{1}+a_{2}+a_{3}+a_{4}+\ldots+a_{n}$

$$
=\left(\frac{a_{3}}{3}-a_{2}\right)+\left(\frac{a_{4}}{3}-a_{3}\right)+\left(\frac{a_{5}}{3}-a_{4}\right)+\left(\frac{a_{6}}{3}-a_{5}\right)+\ldots+\left(\frac{a_{n+1}}{3}-a_{n}\right)+\left(\frac{a_{n+2}}{3}-a_{n+1}\right)
$$

[Dizinin özelliğinden; $a_{n+2}=3 .\left(a_{n+1}+a_{n}\right)$, dolayısıyla $a_{n}=\frac{a_{n+2}}{3}-a_{n+1}$ olur.]

$$
\begin{aligned}
& A=-a_{2}-\frac{2 \cdot a_{3}}{3}-\frac{2 \cdot a_{4}}{3}-\frac{2 \cdot a_{5}}{3}-\ldots-\frac{2 \cdot a_{n}}{3}-\frac{2 \cdot a_{n+1}}{3}+\frac{a_{n+2}}{3} \\
& A=\frac{-a_{2}-2 \cdot\left(a_{2}+a_{3}+a_{4}+\ldots+a_{n}\right)}{3}-\frac{2 \cdot a_{n+1}}{3}+\frac{a_{n+2}}{3} \\
& A=\frac{-a_{2}-2 \cdot\left(A-a_{1}\right)}{3}-\frac{2 \cdot a_{n+1}}{3}+\frac{a_{n+2}}{3}
\end{aligned}
$$




$$
\begin{aligned}
& A=\frac{-3-2 \cdot A+2}{3}-\frac{2 \cdot a_{n+1}}{3}+\frac{a_{n+2}}{3} \quad\left[a_{1}=1 \text { ve } a_{2}=3 \text { olduğundan }\right] \\
& 3 . A=-2 \cdot A+a_{n+2}-2 \cdot a_{n+1}-1 \rightarrow A=\frac{a_{n+2}-2 \cdot a_{n+1}-1}{5} \\
& \text { 2) } a_{1}+a_{3}+a_{5}+\ldots+a_{2 n-1}=\frac{3 \cdot\left(a_{2 n}-2 \cdot a_{2 n-1}\right)+2}{5}
\end{aligned}
$$

İspat: $a_{1}+a_{3}+a_{5}+\ldots+a_{2 n-1}$

$$
\begin{aligned}
& =a_{1}+3 \cdot\left(a_{1}+a_{2}\right)+3 \cdot\left(a_{3}+a_{4}\right)+\ldots+3 \cdot\left(a_{2 n-2}+a_{2 n-3}\right) \\
& =a_{1}+3 \cdot\left(a_{1}+a_{2}+a_{3}+a_{4}+\ldots+a_{2 n-2}\right)=a_{1}+3 \cdot\left(\frac{a_{2 n}-2 \cdot a_{2 n-1}-1}{5}\right)
\end{aligned}
$$

[1. özellikte $n$ yerine $2 n-2$ aldık.]

$$
=\frac{3 \cdot\left(a_{2 n}-2 \cdot a_{2 n-1}\right)+2}{5} \quad\left[a_{1}=1 \text { olarak yerine yazdik. }\right]
$$

3) $a_{2}+a_{4}+a_{6}+\ldots+a_{2 n}=\frac{3 \cdot\left(a_{2 n+1}-2 \cdot a_{2 n}-1\right)}{5}$

İspat: $a_{2}+a_{4}+a_{6}+\ldots+a_{2 n}$

$$
\begin{aligned}
& =a_{2}+3 \cdot\left(a_{2}+a_{3}\right)+3 \cdot\left(a_{4}+a_{5}\right)+\ldots+3 \cdot\left(a_{2 n-2}+a_{2 n-1}\right) \\
& =a_{2}+3 \cdot\left(a_{2}+a_{3}+a_{4}+a_{5}+\ldots+a_{2 n-1}\right)=a_{2}+3 \cdot\left(\frac{a_{2 n+1}-2 \cdot a_{2 n}-1}{5}-1\right)
\end{aligned}
$$

[1. özellikte $n$ yerine $2 n-1$ aldık ve $a_{1}$ değeri 1 'i çıkardık.]

$$
=\frac{3 \cdot\left(a_{2 n+1}-2 \cdot a_{2 n}-1\right)}{5} \quad\left[a_{2}=3 \text { olarak yerine yazdık ve düzenledik. }\right]
$$

4) $a_{1}-a_{2}+a_{3}-a_{4}+\ldots+(-1)^{n+1} \cdot a_{n}=(-1)^{n+1} \cdot 3 \cdot a_{n-1}+1$

İspat: 2. ve 3. özelliklerdeki eşitlikleri taraf tarafa çıkaralım:

$$
\begin{aligned}
& a_{1}+a_{3}+a_{5}+\ldots+a_{2 n-1}=\frac{3 \cdot\left(a_{2 n}-2 \cdot a_{2 n-1}\right)+2}{5} \\
& a_{2}+a_{4}+a_{6}+\ldots+a_{2 n}=\frac{3 \cdot\left(a_{2 n+1}-2 \cdot a_{2 n}-1\right)}{5} \\
& a_{1}-a_{2}+a_{3}-a_{4}+\ldots+a_{2 n-1}-a_{2 n}=\frac{3 \cdot\left(a_{2 n}-2 \cdot a_{2 n-1}\right)+2}{5}-\frac{3 \cdot\left(a_{2 n+1}-2 \cdot a_{2 n}-1\right)}{5} \\
& =\frac{3 \cdot a_{2 n}-6 \cdot a_{2 n-1}+2-3 \cdot a_{2 n+1}+6 \cdot a_{2 n}+3}{5}=\frac{9 \cdot a_{2 n}-6 \cdot a_{2 n-1}-3 \cdot a_{2 n+1}+5}{5} \\
& {\left[a_{2 n+1}=3 \cdot a_{2 n}+3 \cdot a_{2 n-1} \text { yazdik. }\right]}
\end{aligned}
$$


$=\frac{9 \cdot a_{2 n}-6 \cdot a_{2 n-1}-9 \cdot a_{2 n}-9 \cdot a_{2 n-1}+5}{5}=\frac{-15 \cdot a_{2 n-1}+5}{5}$

$=\frac{-15 \cdot a_{2 n-1}+5}{5}=\mathbf{1}-\mathbf{3} \cdot a_{2 n-1}$

Son eşitlikte tek indisli terimlerin pozitif katsayılı, çift indisli terimlerin negatif katsayılı olduğuna dikkat ederek ve $2 \mathrm{n}$ yerine $n$ yazılarak düzenlenirse aşağıdaki eşitlik elde edilir:

$a_{1}-a_{2}+a_{3}-a_{4}+\ldots+(-1)^{n+1} \cdot a_{n}=(-1)^{n+1} \cdot 3 \cdot a_{n-1}+1$

5) $a_{n+m}=3 \cdot a_{n-1} \cdot a_{m}+a_{n} \cdot a_{m+1}$

İspat: m’ye göre tümevarım yapalım.

$\mathrm{m}=1$ için doğru mu? $\quad a_{n+1}=3 \cdot a_{n-1} \cdot a_{1}+a_{n} \cdot a_{1+1} \rightarrow a_{n+1}=3 \cdot a_{n-1}+3 \cdot a_{n} \quad\left[a_{1}=1\right.$ ve $\left.a_{2}=3\right]$ doğrudur

$\mathrm{m}=\mathrm{k}$ için, $\quad a_{n+k}=3 \cdot a_{n-1} \cdot a_{k}+a_{n} \cdot a_{k+1}$ eşitliği doğru olsun.

$\mathrm{m}=\mathrm{k}+1$ için doğru olur $\mathrm{mu}$ ?

$a_{n+(k+1)}=a_{(n+1)+k}=3 \cdot a_{n} \cdot a_{k}+a_{n+1} \cdot a_{k+1} \quad[\mathrm{~m}=\mathrm{k}$ için doğru demiştik. $]$

$=3 \cdot a_{n} \cdot a_{k}+\left(3 \cdot a_{n}+3 \cdot a_{n-1}\right) \cdot a_{k+1}=3 \cdot a_{n} \cdot a_{k}+3 \cdot a_{n} \cdot a_{k+1}+3 \cdot a_{n-1} \cdot a_{k+1}$

$=a_{n} \cdot\left(3 \cdot a_{k}+3 \cdot a_{k+1}\right)+3 \cdot a_{n-1} \cdot a_{k+1}=a_{n} \cdot a_{k+2}+3 \cdot a_{n-1} \cdot a_{k+1}$

$\mathrm{m}=\mathrm{k}+1$ için doğru oldu. Öyleyse özellik, dizinin tüm terimleri için geçerlidir.

6) $a_{2 n}=\frac{\left(a_{n+1}\right)^{2}-\left(3 \cdot a_{n-1}\right)^{2}}{3}$

İspat: 5. özellikteki eşitlikte $\mathrm{m}=\mathrm{n}$ alalım. $\quad a_{n+n}=3 \cdot a_{n-1} \cdot a_{n}+a_{n} \cdot a_{n+1}$

$$
\begin{aligned}
& a_{2 n}=3 \cdot a_{n-1} \cdot\left(\frac{a_{n+1}-3 \cdot a_{n-1}}{3}\right)+\left(\frac{a_{n+1}-3 \cdot a_{n-1}}{3}\right) \cdot a_{n+1} \\
& a_{2 n}=\frac{3 \cdot a_{n-1} \cdot a_{n+1}-\left(3 \cdot a_{n-1}\right)^{2}+\left(a_{n+1}\right)^{2}-3 \cdot a_{n-1} \cdot a_{n+1}}{3} \\
& a_{2 n}=\frac{\left(a_{n+1}\right)^{2}-\left(3 \cdot a_{n-1}\right)^{2}}{3}
\end{aligned}
$$


Bulgular, özelliklerin karşılaştırmalı görülebilmesi için Çizelge-1'de özetlenmiştir.

\begin{tabular}{|c|c|c|c|c|c|c|c|}
\hline Özellik & Fibonacci & $1 K(k=2)$ & $1 K(k=3)$ & K1 (k=2) & K1 (k=3) & $K K(k=2)$ & KK (k=3) \\
\hline$a_{1}+a_{2}+a_{3}+\ldots+a_{n}$ & $a_{n+2}-1$ & $\frac{a_{n+1}+a_{n}-1}{2}$ & $\frac{a_{n+1}+a_{n}-1}{3}$ & $\frac{a_{n+2}-1}{2}$ & $\frac{a_{n+2}-1}{3}$ & $\frac{a_{n+2}-a_{n+1}-1}{3}$ & $\frac{a_{n+2}-2 \cdot a_{n+1}-1}{5}$ \\
\hline$a_{1}+a_{3}+a_{5}+\ldots+a_{2 n-1}$ & $a_{2 n}$ & $\frac{a_{2 n}}{2}$ & $\frac{a_{2 n}}{3}$ & $\frac{a_{2 n+1}+n-1}{3}$ & $\frac{3 \cdot a_{2 n+1}-a_{2 n+2}-2}{3}$ & $\frac{1+2 .\left(a_{2 n}-a_{2 n-1}\right)}{3}$ & $\frac{3 \cdot\left(a_{2 n}-2 \cdot a_{2 n-1}\right)+2}{5}$ \\
\hline$a_{2}+a_{4}+a_{6}+\ldots+a_{2 n}$ & $a_{2 n+1}-1$ & $\frac{a_{2 n+1}-1}{2}$ & $\frac{a_{2 n+1}-1}{3}$ & $\frac{a_{2(n+1)}-n-1}{3}$ & $\frac{2 \cdot a_{2 n+2}-3 \cdot a_{2 n+1}+1}{3}$ & $\frac{2 .\left(a_{2 n+1}-a_{2 n}-1\right)}{3}$ & $\frac{3 \cdot\left(a_{2 n+1}-2 \cdot a_{2 n}-1\right)}{5}$ \\
\hline$a_{1}-a_{2}+a_{3}-\ldots+(-1)^{n+1} \cdot a_{n}$ & $(-1)^{n+1} \cdot a_{n-1}+1$ & $\frac{(-1)^{n}\left[a_{n}-a_{n+1}\right]+1}{2}$ & $\frac{(-1)^{n}\left[a_{n}-a_{n+1}\right]+1}{3}$ & $\frac{(-1)^{n} \cdot\left[a_{n+1}-a_{n+2}\right]+n}{3}$ & $(-1)^{n} \cdot\left[2 \cdot a_{n+1}-a_{n+2}\right]-1$ & $(-1)^{n+1} \cdot 2 \cdot a_{n-1}+1$ & $(-1)^{n+1} \cdot 3 \cdot a_{n-1}+1$ \\
\hline$a_{1}^{2}+a_{2}^{2}+a_{3}^{2}+\ldots+a_{n}^{2}$ & $a_{n} \cdot a_{n+1}$ & $\frac{a_{n} \cdot a_{n+1}}{2}$ & $\frac{a_{n} \cdot a_{n+1}}{3}$ & $\frac{(-1)^{n+1} \cdot a_{n+3}+3 \cdot a_{n+1}^{2}+n}{9}$ & - & - & - \\
\hline$a_{n+m}$ & $a_{n-1} \cdot a_{m}+a_{n} \cdot a_{m+1}$ & $a_{n-1} \cdot a_{m}+a_{n} \cdot a_{m+1}$ & $a_{n-1} \cdot a_{m}+a_{n} \cdot a_{m+1}$ & 2. $a_{n-1} \cdot a_{m}+a_{n} \cdot a_{m+1}$ & 3. $a_{n-1} \cdot a_{m}+a_{n} \cdot a_{m+1}$ & 2. $a_{n-1} \cdot a_{m}+a_{n} \cdot a_{m+1}$ & 3. $a_{n-1} \cdot a_{m}+a_{n} \cdot a_{m+1}$ \\
\hline$a_{2 n}$ & $a_{n+1}^{2}-a_{n-1}^{2}$ & $\frac{\left(a_{n+1}^{2}-a_{n-1}^{2}\right)}{2}$ & $\frac{\left(a_{n+1}^{2}-a_{n-1}^{2}\right)}{3}$ & $\left(a_{n+1}\right)^{2}-\left(2 \cdot a_{n-1}\right)^{2}$ & $\left(a_{n+1}\right)^{2}-\left(3 \cdot a_{n-1}\right)^{2}$ & $\frac{\left(a_{n+1}\right)^{2}-\left(2 \cdot a_{n-1}\right)^{2}}{2}$ & $\frac{\left(a_{n+1}\right)^{2}-\left(3 \cdot a_{n-1}\right)^{2}}{3}$ \\
\hline
\end{tabular}

\section{SONUÇ ve TARTIŞMA}

Fibonacci dizisi için bilinen 7 temel özelliğin benzerlerinin, bu çalışmada $1 \mathrm{~K}, \mathrm{~K} 1$ ve KK diye isimlendirilen dizi ailelerinin elamanı olan dizilerde mevcut olup olmadığ 1 a raştırılmıştır. Her bir aileden ilk iki dizi üzerinde yani toplam 6 dizi üzerinde çalışılmıştır. $1 \mathrm{~K}$ ailesinden alınan iki dizi için de bahsedilen 7 özellik elde edilmiştir. K1 ailesinden alınan ilk dizi ( $k=2$ durumu) için, temel 7 özelliğin yanı sıra bu özelliklerin ispatlanmasına yardımcı olacak bir özellik ve bu özelliğin sonucu olan 2 özellik olmak üzere toplam 10 özellik ispatlarıyla verilmiştir. K1 ailesinden alınan ikinci dizi $(\mathrm{k}=3$ durumu) için, temel özelliklerden terimlerin kareleri toplamıyla ilgili olan özellik hariç 6 özellik ispatlarıyla verilmiştir. KK ailesinden alınan iki dizi için de yine temel özelliklerden terimlerin kareleri toplamıla ilgili olan özellik hariç 6'şar özellik ispatlarıyla verilmiştir. Böylece, Fibonacci dizisinin mantığına benzer şekilde üretilen dizilerle ilgili Fibonacci dizisinin özelliklerine benzeyen toplam 42 özellik ispatlarıyla verilmiştir.

Bulunan özelliklere bakıldı̆̆ında, hem aynı aile içindeki dizilerin kendi aralarında hem de aileler arasında benzerlikler, ilişkiler görülmektedir. $\mathrm{Bu}$ sayede özellikler genelleştirilebilir ve bir sonraki adımda özelliğin ne şekilde değişeceği öngörülebilir. Örneğin tek sayı indisli terimlerin toplamını veren ifade, $1 \mathrm{~K}$ dizi ailesinde $\mathrm{k}=2$ için oluşturulan dizide $\frac{a_{2 n}}{2}$ iken; aynı ailedeki $\mathrm{k}=3$ için oluşturulan dizide $\frac{a_{2 n}}{3}$ olmuştur. Bu şekilde aynı ailedeki sonraki diziler için $\frac{a_{2 n}}{4}, \frac{a_{2 n}}{5}, \ldots$ şeklinde gidebileceği tahmin edilebilir.

Literatürdeki çalışmalara bakıldı̆̆ında bu çalışmadaki yöntem ve bulgularla benzerlik gösteren bir çalışmayla karşılaşılmamıştır. Altun (2016), Güleç (2014) ve Bolat (2008) da çalışmalarını Fibonacci dizine benzer şekilde türetilen dizilerle yapmalarına rağmen onlar çok daha farklı dizi aileleriyle, farklı yön ve yöntemlerle çalışmışlardır. Yalnızca Köken'in (2008) yaptı̆̆ çalışmadaki K1 ailesinin k=2 için elde edilen dizisi olan Jacobsthal dizisinin bazı özelliklerinden bahsedilmiş ancak tamamen farklı bir yol izlenmiştir.

Gelecek çalışmalarda bu çalışmada ele alınan dizi ailelerinin diğer elemanlarında $(\mathrm{k}=4,5, \ldots) \mathrm{da}$ benzer özellikler araştırılabilir. Bulunan özellikler dışında dizilerin bölünebilme özellikleri gibi yeni özellikler araştırılabilir. Bazı diziler için bulunamayan terimlerin kareleri toplamiyla ilgili olan özellik üzerinde çalışılabilir. Yine Fibonacci dizisine benzer şekilde farklı diziler oluşturulup, bu dizlerin özellikleri ispatlanabilir. Örneğin, bir terimi kendinden önceki iki terimin farklı tam say 1 kuvvetlerinin toplamından oluşan diziler ele alınıp incelenebilir. Bu çalışmada elde edilen özelliklerin, uygulama alanları araştırılabilir, mümkünse anlamları analitik olarak yeniden yorumlanabilir. 


\section{KAYNAKLAR}

Altun,İ. (2016). "Genelleştirilmiş Fibonacci ve Lucas Polinomlarında Yeni Bir Aile". Yüksek Lisans Tezi, Erzincan Üniversitesi, Erzincan-Türkiye.

Argün, Z., Arıkan, A., Bulut, S., Halıcıoğlu, S. (2014). Temel Matematik Kavramlarının Künyesi: Gazi Kitabevi. Ankara-Türkiye.

Bolat, C. (2008). "k-Fibonacci, k-Lucas Sayılarının Özellikleri ve Uygulamaları". Yüksek Lisans Tezi, Selçuk Üniversitesi. Konya-Türkiye.

Güleç,H.H. (2014). "Pell Matris Dizileri ve Özellikleri". Doktora Tezi, Selçuk Üniversitesi. Konya-Türkiye.
Köken, F. (2008). "Jacobsthal ve Jacobsthal Lucas Sayılarının Özelllikleri ve Uygulamaları". Yüksek Lisans Tezi, Selçuk Üniversitesi. Konya-Türkiye.

Maksudov, F. ve Veliev, C. (1993) "Fibonacci sayıları hakkında bir kural". Matematik Dünyası Dergisi, 4: 14 Erişim adresi: http://www.matematikdunyasi. org / arsiv/ P D F_eskis ayilar / 92 _ 4 _ $144_{-} 16$ _ FIBONACCI.pdf Son Erişim Tarihi: 02.10.2018.

Pappas, T. (2007). Yaşayan Matematik: Doruk Yayıncılık. İstanbul-Türkiye. 